\title{
Enhanced Bandwidth Allocation Technique and Protocol Standards to Improve QoS in Internet of Things
}

\author{
R. Mohandas, D. John Aravindhar, D. Praveenkumar
}

\begin{abstract}
Internet of Things (IoT) is efficiently plays vital role in development of several sectors by offering many opportunities to grow the economy and improve the life standard through connecting billions of "Things" which provides business opportunities in different sectors and encounter many technical and application challenges. This paper emphasizes the role of Dynamic bandwidth allocation and protocols standards in various IoT sectors such as healthcare, education, agriculture, industrial, transportation, smart cities etc., and focuses on the challenges in providing uninterrupted bandwidth to all IoT devices with existing infrastructure, which depends on standardized protocols and network devices to establish connection with heterogeneous IoT devices. This paper covers Enhanced Dynamic Bandwidth Techniques, protocol standards and policies in IoT network technologies to Improve QoS in IoT devices.
\end{abstract}

Index Terms--- BLE, IoT, NFC, RFID, Zigbee, EBAT, QoS.

\section{INTRODUCTION}

The Internet of Things (IoT) is the network of connected things, a "Thing" is any device or object, embedded with electronic sensors able to transfer data in networks without human interaction, examples are health monitor, vehicle tracking adaptor, smart parking sensors, devices in smart cities [1].IoT focuses at making the Internet even more immersive and ubiquitous. Further enabling easy communication and access among variety of devices such as, heart monitor, security cameras, home automation, and smart lock and so on, the IoT will stand-in the increasing of applications that make use of the potentially massive amount and variety of data generated by such objects to provide new services to people, companies, and government [2].Government initiative towards smart city and eGovernance supported by technological enhancement in computing power, battery capacities and storage, available at economical. This tendency is enabling the development of small-scale electronic devices with computing capabilities such as communication, identification and transmission, which can be embedded in other systems, devices, and facilities [3].

Currently, in India, there are at least 130 million interconnected devices; it is predictable to reach 7billion devices by 2020 . The market volume of IoT in the country

Revised Manuscript Received on 14, October 2019.

R. Mohandas, Assistant Professor (SG), Department of CSE, Hindustan Institute of Technology \& Science, Chennai, India. (e-mail: rmohandas@hindustanuniv.ac.in)

Dr.D. John Aravindhar, Professor, Department of CSE, Department of CSE, Hindustan Institute of Technology \& Science, Chennai, India.(email: jaravindhar@hindustanuniv.ac.in)

D. Praveenkumar, Assistant Professor, Department of CSE, Hindustan Institute of Technology \& Science, Chennai, India. (e-mail: dpkumar@hindustanuniv.ac.in) expected move 5 times folds from $\$ 1.2$ billion to $\$ 7$ billion by 2022 [4]

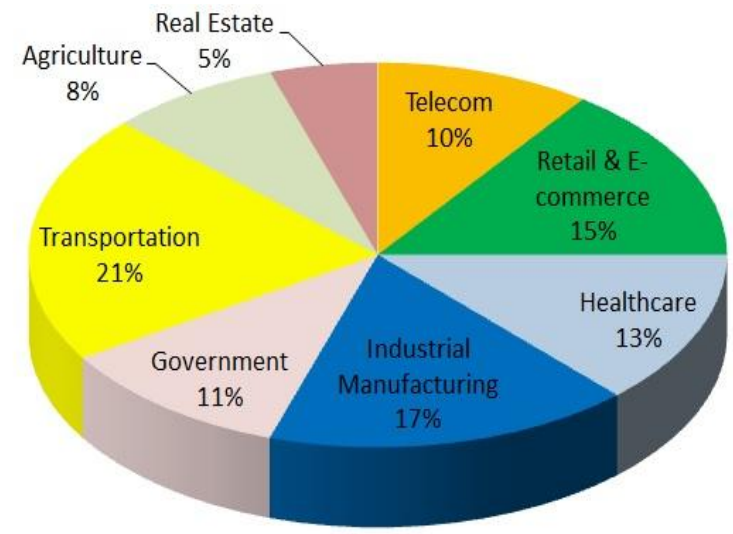

Figure 1: IoT application in Across Industries

This paper presented as follows. Section II- presents opportunity and benefits of IoT. Section III-presents R\&D policy and standardization. Section IV- interoperable infrastructure and architecture. Section V-Role of IoT in national development. Section VI-IoT Clustering, Section VII-Enhanced Bandwidth Allocation Technique. Section VIII- IoT Challenges, Section IX-conclusion, Section XReferences.

\section{OPPORTUNITY AND BENEFITS OF IOT}

The demand for connected devices, vehicles and products is accelerating towards enormous point. The following factors catalyst IoT market.

Supplier attention: Surging demand on IoT kits has led, tech Giants such as Microsoft, IBM, Intel, Qualcomm companies to produce IoT tools and products.

Technological developments: The growth of IoT has increased due to the drop in cost of smart phones and components like sensors, lure the semiconductor companies to produce IoT products for various use cases and thus increased the corporate and government to invest in IoT and related opportunities (e.g. Smart cities, Digital India)

Growing demand: Demand for the first generation of Internet of Things devices such as smart watches, fitness bands, and smart thermostats will boost as integral technologies advance and their costs fall. A similar dynamic will occur with the rise of usage of IoT devices in healthcare, retail, industrial, smart home applications, and transportation, entertainment and agriculture sectors.

\section{Published By:}

Blue Eyes Intelligence Engineerin 

IN INTERNET OF THINGS

\section{RESEARCH \& DEVELOPMENT, POLICY AND STANDARDIZATION}

\section{Research and Development}

Identifying expert and potential members for R\&D field, enables cloud based projects for IoT research initiatives. To create research labs for hardware to hardware and hardware to software alliance encourages private sectors to invest in research and development and to commence IoT related projects with international partners. An innovative project named "International IoT Research Collaboration scheme (IIRC)" is started by Deit Y, Government of India in support with an affiliated association/supporting body with the following objectives: a. Under IIRC proposal, government will team up and initiate treaties with other countries to generate joint projects for research and development in IoT on $40 \%$ share. b. IIRC proposal will spend fund to IoT industry for approved projects after analyzing the competences of the bidder, finally approves loan and offer equity and grand, c. Under IIRC proposal, the selected organization will handle the whole process from Call for Proposal to project closures.

\section{Policies}

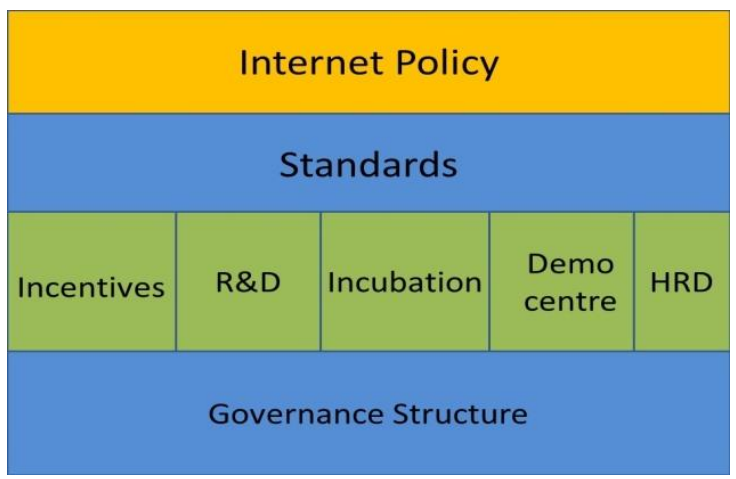

Figure 2: IoT policy and standards

The Policy structure of the IoT has been recommended to be implemented via a multi-pillar method. The method comprises of five vertical pillars: Incentives, $R \& D$, Incubation Demo centre, Human Resource Development Engagements and Standards

\section{Standards}

To achieve the economic benefits and safe interoperability, especially among systems is depends on success in regulating the IoT standard and connections. There are such standards in progress for example, LPWAN-a Low Power Wide Area Network blueprint proposed for wireless battery- covers the area such as global, national and regional networks - incorporates secure bidirectional communication, localization and mobility services for regular interoperability among smart devices [6].

The IoT system model contains the architecture standards, communication protocol standards, network standards, security standards, the application standards and the information processing standards. To ease supporting standards around IoT technologies, national and international research and industry bodies doing major role such as IoT regulation, spectrum allocation and protocols standards with international quality/integrity standards for data formation and data traceability.
Formulate the core committee to establish open structure for IoT Architecture platform, security and privacy technologies, hybrid networking technologies, hardware's, sensors, actuators, energy harvesting and conversion, silicon and semiconductor manufacturing. CoE-IoT is working with Global networking Standards Committees such as IEEE, ITU and other relevant forums for making IoT standards in India [5].

\section{ARCHITECTURE \& INFRASTRUCTURE}

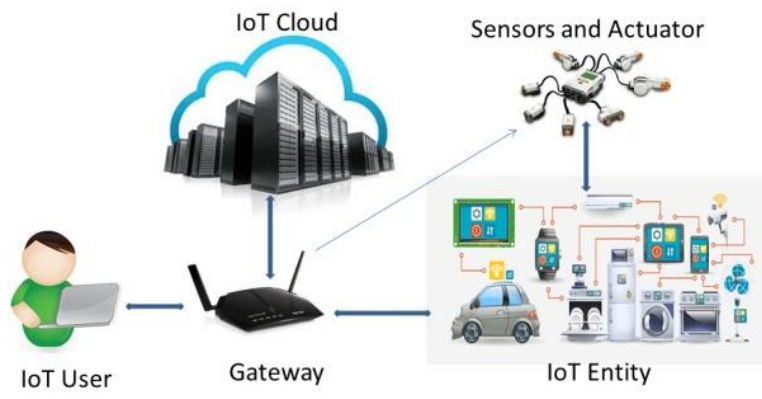

Figure 3: IoT Architecture

The architecture of IoT is framed into five layers. Layer 1: User transfers the control information to IoT entities via Gateway. Layer2: IoT gateways execute several significant functions such as device connectivity, data filtering, processing, security, updating and protocol translation. Layer 3: IoT entities such as wearable, thermostats, vehicles, home appliances, health monitoring device and many more are interconnected with users and cloud via gateways. Layer 4: Sensors perform some input functions by sensing or feeling the physical changes in the characteristics of a system in response to a stimulus, based receiving a control signal, the actuator responds by converting the energy into mechanical motion.

Layer 5: IOT Cloud will generate compatible data every hour and the backend must be able to handle this load very effectively in real time, IoT cloud advances new data driven insights and run actions over IoT devices without any human intervention via data analytics driven by machine intelligence at cloud.

\section{IoT Infrastructure}

The Internet of Things needs a strong pillar for connecting devices; wider adaption needs right backbone and proper infrastructure, which needs an intelligently connected and securely designed Sensing, Computing and Storage Infrastructure which makes the infrastructure robust and flexile for IoT solutions.

Platforms: The IoT is directed at connecting devices with each other. A standard platform is built to provide the basic layer of services with interoperability between devices, cloud services and basic IP networking, application layer security, and device management. 


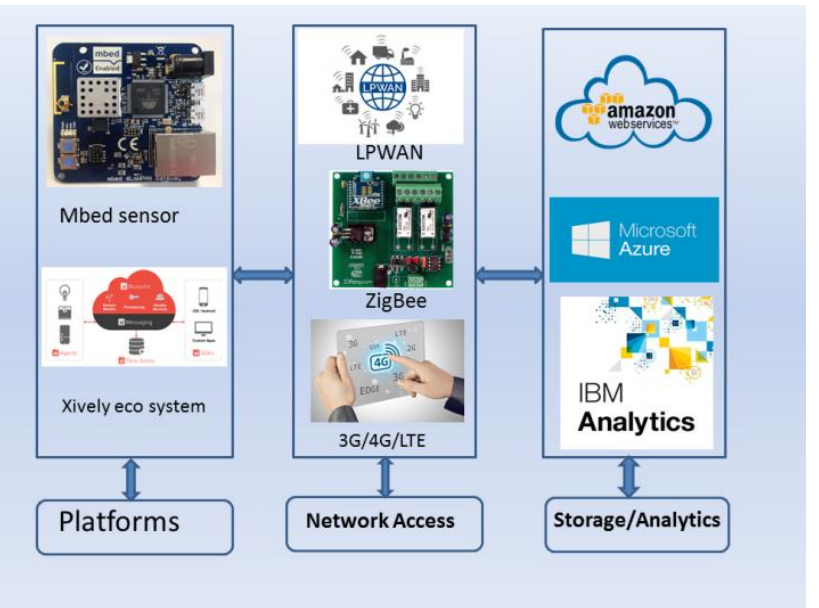

Figure 4: IoT Platforms

Network Access: Networking is the primary role of IoT for connection of devices and the transmission of data. Many of the IoT applications use diverse specifications for communication and data transfer which need to be operated even in low power; hence IoT space is adopted with advanced networking technologies and protocols, examples, LPWAN, ZigBEE, Z-Wave, SNAP, EnOcean which serves explicit profile of power usage, bandwidth, and range.

Storage and Analytics: The flow of data that comes from IoT devices need to be stored securely atIoT cloud services. Various Tech giants such as Microsoft, Amazon, IBM are expanding their business from cloud to IoT and provides the services of forecasting, predictive, streaming and data analytics to enable the IoT applications to take smart decisions without human intervention [7].

\section{IOT ROLE IN NATIONS' DEVELOPMENT}

Smart city: Mission of smart cities have the objectives of afford state of art infrastructure, clean and viable environment and standard life style to its. The smart city would have primary infrastructure fundamentals such as: i. Assured electricity supply ii. Adequate water supply iii. Efficient urban mobility and public transport. iv. Sanitation, including solid waste management v. Robust IT connectivity and digitalization, vi. Compacts house. vii. Good administration with each citizen participation, eGovernance, viii .Maintainable environment ix. Health and education x. Safety and security for each citizen, city's physical infrastructure and services with the latest technological offered by IoT, plays the major role in seal the individual blocks of smart cities.

Security: Smart city security is ensured through IoT surveillance cameras which act as a virtual gate at the entrance and all the areas of smart city.Energy: IoT encourages energy harvesting via renewable energy. Each home is equipped with solar panels with a storage battery and monitor, to show the residents how much energy is being used on a daily basis. In addition, the residents can sell the surplus energy back to the national grid.

\section{Digital Transformation}

Government proposes digital transformation in all sectors, where IoT is an important tool to achieve the digital economy - IoT empowers the citizens to access the basic governance services like health monitoring in rural areas, education to all mission, finance assistance to entrepreneur and farmers, ease of electronic transaction makes the all the sector to adopt paperless movement, which results in cashless transaction, support the movement of saves the document in governments digi locker, where people can view and share the data safe and secure way, to achieve this government builds optimized cloud infrastructure for digital transformation. The primary focus of digital transformation movement is to deliver digital infrastructure service to all area, so that basic amenities such as health, education, government schemes and financial aid can reach all citizens [8]. The Digital transformation program escalates reach of digital infrastructure with the aim of enabling electronic delivery of government facilities to citizens. The power of Digital transformation is providing an ecosystem in which every citizen is digitally empowered and has the key to access electronic services [9].
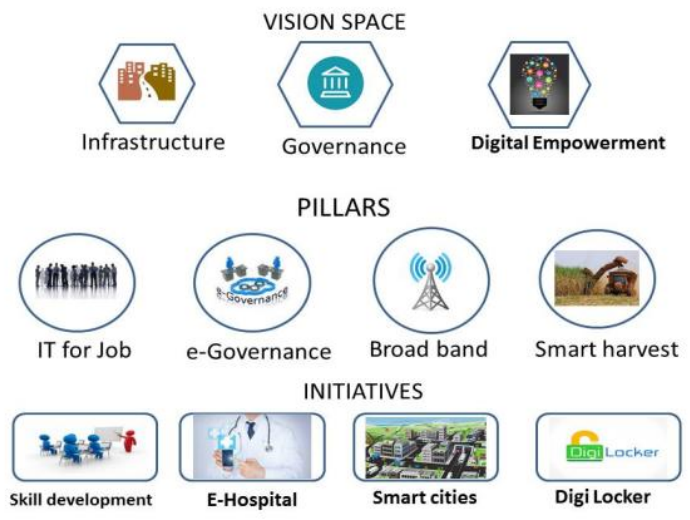

Figure 5: Overview of Digital transformation

Transportation: IoT empowered cars are dominant in automobile industry, providing the services of traffic alerts, emergency roadside assistance, parts maintenance alerts, monitor and process real-time traffic data to help manage transportation infrastructures, assess road conditions and ease congestion, using real-time data and alerts to optimize routes in public transport, monitor and quickly respond to delays or issues as they occur in IoT enabled vehicles. Automobile industry expects there will be over 270 million IoT enabled vehicles on the road by 2019 [10].

Agriculture: It is expected to 70 million IoT devices and sensor will be utilized in the agriculture field by 2020 . IoT devices will be able to provide information on rainfall, pest infestation, and soil nutrition through IoT sensors. Farmers increase the crop yields based on the input data obtained from the IoT sensors placed in the farm filed to get various data such as temperature and acidity levels in the soil and other variables from the field [11].

Retail: IoT enabled devices are being used in stores to promptly scan and call up product information, see reviews in social media over smart phones. Smart shelves in retail store detects when the inventory is getting decreased. IoT technology work independently to aid stock replenishment and IoT sensors observes freshness of perishable goods. Retail industry predicts more revenues through generated messages based on customer interest data which is gathered from various stores and analyzed in cloud [12]. 


\section{ENHANCED BANDWIDTH ALLOCATION TECHNIQUE AND PROTOCOL STANDARDS TO IMPROVE QOS IN INTERNET OF THINGS}

Healthcare: IoT paves the platform to improve the health index of citizens by regulating the infrastructure of healthcare to deliver telemedicine facilities to all areas in the country, which facilitate remote area people to access video consultation facility with physician in the city hospitals. Automatic identification, tracking of people and biomedical devices in hospitals are being automated using wireless sensor network (WSN), radio frequency identification (RFID), ultra-high-frequency (UHF) and smart mobile technologies [13].

\section{IOT CLUSTERING METHOD}

The IoT clusters formed based on its bandwidth usage pattern .The bandwidth Clustering-planning approach using unsupervised learning targeted to group similar bandwidth utilization devices for bandwidth allocation in standard rate. The unsupervised learning observes the bandwidth usage over the period with the utilization pattern, the cluster range can formed then the nodes that have a close identity with the cluster range can added together based on type of application, severe care and using pattern such an idea, clusters type can build in the midst of nodes $\mathrm{N}$ [14].

Enhance a system to improve the functionalities of existing clustering system, which can track the patterns of the connected IoT devices, generate a usage pattern, and even record on the cloud. In this paper, IDBA method applied over bandwidth management to improve the quality of IoT devices, which, projects the idle, medium or severe level in it usage level. Once the system gets adapted to the environment in first few initial attempts, then any change in the level of usage will updated on cloud systems.

Proposed method of this paper characterized as

Dedicated bandwidth allocated to IoT devices based on cluster category

Normal bandwidth to group of IoT devices, which send and receive data occasionally. Guaranteed bandwidth allocated to the IoT devices where system cannot tolerate bandwidth breakage. Bandwidth management policy applied to all the connected devices, as per policy table devices receives bandwidth. a bandwidth effectiveness function,

Table 1: Government of India Initiative to build ICT infrastructure for Digital Transformation

\begin{tabular}{|c|c|c|}
\hline Initiative & Action Plan & Present Position \\
\hline Smart Cities & $\begin{array}{l}\text { INR } 6 \text { billion allotted to each city over } 5 \text { years } \\
\text { towards the purpose of Establishing of } 107 \text { smart } \\
\text { cities by } 2020 \text {. }\end{array}$ & $\begin{array}{l}\checkmark \checkmark \text { Under the smart city mission } 50 \text { cities have been } \\
\text { selected Allotment of INR } 30 \text { billion in central budget } \\
2016-17 \text {. } \\
\checkmark \quad \text { in the } 2015-16 \text { central budget fund allotment of INR } \\
75 \text { billion has been revised to INR } 1.5 \text { billion }\end{array}$ \\
\hline BharatNet & $\begin{array}{l}\text { high speed Fiber Optical Cable } \\
\text { is laid to offer broadband connection to } 2,60,000 \\
\text { Gram Panchayats }\end{array}$ & $\begin{array}{l}\text { Fiber optic cable laid of } 1,50,370 \mathrm{~km} \text { OFC to } 65,457 \\
\text { GPs connectivity done. }\end{array}$ \\
\hline $\begin{array}{l}\text { Public Wi-Fi } \\
\text { Hotspots }\end{array}$ & $\begin{array}{l}\text { Empower citizens to access free internet data by } \\
\text { Construction of public Wi-Fi hotspots in India }\end{array}$ & $\begin{array}{ll}\checkmark & 35,000 \text { Wi-Fi hotspots are currently available in India } \\
\checkmark & \text { To encounter the worldwide average of one hotspot } \\
\text { for every citizen India must have } 10 \text { million Wi-Fi } \\
\text { hotspot }\end{array}$ \\
\hline $\begin{array}{l}\text { Global Access } \\
\text { to Mobile }\end{array}$ & $\begin{array}{l}\text { Target of providing mobile coverage access to } \\
55,600 \text { villages }\end{array}$ & Planned to achieve the target before Feb 2018. \\
\hline
\end{tabular}

(Source: Deloitte)[9].
$E(x)$, that describes the user's preference foran allocation of $x$ bandwidth units.

\section{PSEUDO-CODE REPRESENTATION ENHANCED BANDWIDTH ALLOCATION TECHNIQUES (EBAT)}

(1) Setup WholeBn

$m=0, \forall m \in m i$

(2) Eliminateagainst the link set Nall links NENthat havea amount of contacts crossing them pnequivalent to 0

(3) Each linkn $\in N$, calculate $H n=T n / p n$

(4) Ascertain the path $\beta$ that reduces $H \beta$

i.e. $\beta \mid H \beta=\min M(H m)$

(5) Fixedhp $m=H \beta, \forall m \in M \beta$, where $M \beta \subseteq M i$ is the established desirouspaths that cross connection $\beta$ (6)Each noden, inform the enduringcapacity and the sum of overpassgraspingnetworks as follows:Tn $=T_{n}{ }^{-} \_m \in n \beta \mathrm{hpl}$

- cn m

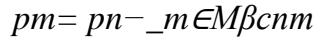

(8) Eliminate from agreedNNodeßand nodes that have $p m=$ 0

(8) IfNis blank, then halt; else drive to Stage (3)

To consider obsessed by description user's weights it is adequate to standby $\mathrm{pm}$. with $y m$, which well defined as the figure of the loads of all desirous link that are routed on node $n$.

It is Necessary to clarify that our techniques can temporarily present some restrictions in bandwidth allocation, and then the bandwidth allocated to devices can utmost twice from an update interval to the succeeding one. This could disturb the performance of nodes that practice sudden surges in their broadcast rate.

In this section, we calculate statistically this effect presenting at the similar time how it compensated by increased network bandwidth in the entire considered network Situations under severe traffic load and protocol standards [15]. 

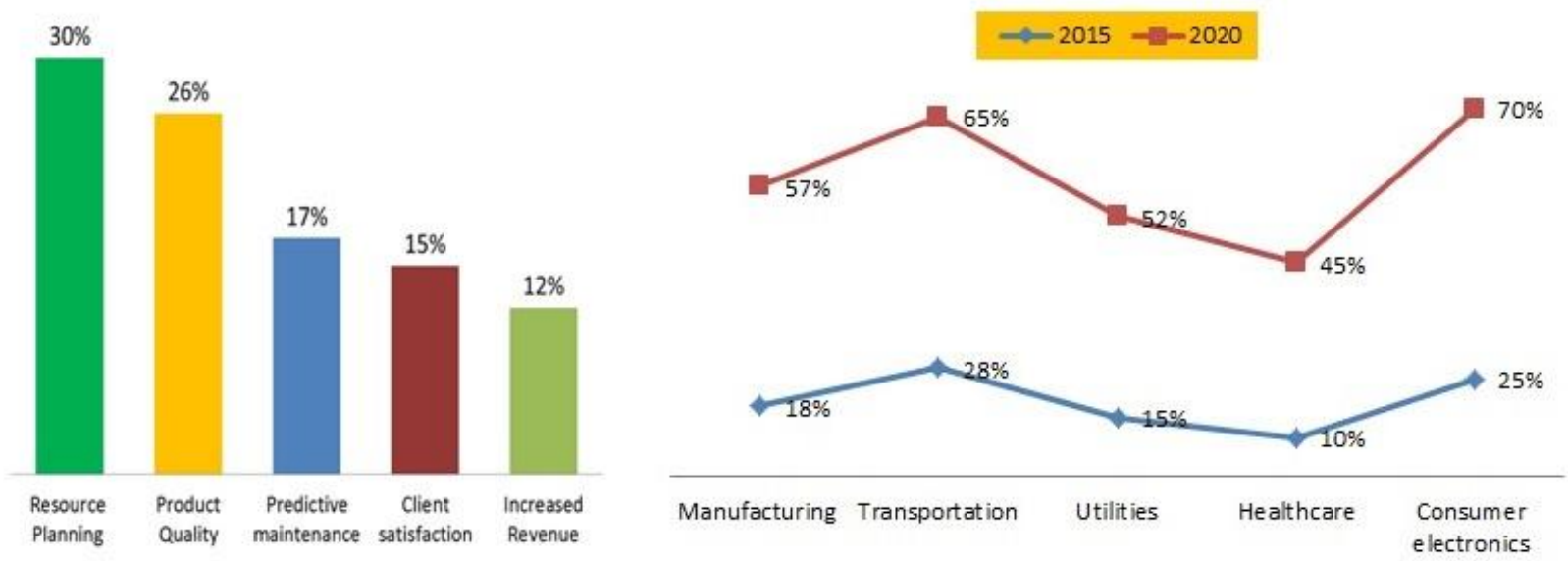

Figure 6: Influence of IoT in Percentages

\section{IOT CHALLENGES \& RESULTS}

Communication is a backbone to the Internet of Things, while implementing the standards the following challenges in networking technologies are inevitable.

\section{Bandwidth}

Service providers take accounts of the following characteristics to enable dynamic bandwidth allocation: group the devices based on data utilization pattern, monitor whether the devices continuously sending data or at an erratic level the idle devices bandwidth can be allotted to the cluster of devices where bandwidth demand is high.

\section{Range}

The network area is named after based on its coverage pattern: PAN, LAN, MAN, WAN. The challenging task lies in selecting appropriate networks and protocol based on the application, example BLE is suitable for room environment where it will inappropriate for agriculture filed.

\section{Power Usage}

Here energy harvesting from solar power, radio frequency and vibration in factory floor is the main source of power. To extend the battery life, set the device into sleep mode when it is idle.

\section{Security}

Ensuring end-to-end security is an important factor in Safeguard IoT devices in the connected environment. Authentication, encryption, and open port protection, message integrity, confidentiality to be ensured by IoT protocols.

\section{CONCLUSION}

IoT creates innovative products and services, drive down operational cost and improves the revenue. Before realizing the benefits of IoT, proper infrastructure and standards needs to establish. This paper gives an analysis of role of Enhanced bandwidth allocation techniques and protocols standards in improving the quality of service in IoT devices. Intelligent bandwidth management policy applied over the cluster based IoT devices. The principal

\section{REFERENCES}

1. https://www.ibm.com/internet-of-things/ resources/ library/ what-is-iot/

2. P. Bellavista, G. Cardone, A. Corradi, and L. Foschini, "Convergence of MANET and WSN in IoT urban scenarios,” IEEE Sens. J., vol. 13, no. 10, pp. 3558-3567, Oct. 2013

3. Shanzhi Chen, HuiXu, Dake Liu, Bo Hu, and Hucheng Wang, "A Vision of IoT: Applications, Challenges, And Opportunities With China Perspective" IEEE INTERNET OF THINGS JOURNAL, VOL. 1, NO. 4, AUGUST 2014

4. http://www.livemint.com/Industry/tF2p8P7BF39r1e1S8Xd 2SJ/Indian-Internet-of-Things-market-value-to-touch-9billion-b.html

5. http://meity.gov.in/sites/upload_files/dit/files/Draft-IoTPolicy\%20(1).pdf

6. Phillip A. Laplante, Jeffrey Voas, Nancy Laplante, "Standards for the Internet of Things: A Case Study in Disaster Response" IEEE Computer Society May 2016

7. https://iot-analytics.com/iot-infrastructure-providers-iothype/

8. http://www.businesstoday.in/opinion/columns/role-ofinternet-of-things-in-india-digital-transformation/story /227415.html

9. https://www2.deloitte.com/content/dam/Deloitte/in/Docum ents/technology-media-telecommunications/in-tmt-digitalindia-unlock-opportunity-noexp.pdf

10. https://www.microsoft.com/en-in/internet-of-things/ transportation

11. http://www.digikey.com/en/articles/techzone/2015/nov/po wering-iot-how-next-generation-of-external-powersupplies-will-help-minimize-energy-consumption

12. R.Mohandas Dr. D. John Aravindhar" A Survey on IoT: Business Perspective Predictions, Trends and Marketing" Advances in natural applied sciences MAY 2017. 
13. Luca Catarinucci, Danilo de Donno, Luca Mainetti, Luca Palano, Luigi Patrono, Maria Laura Stefanizzi, and Luciano Tarricone" An IoT-Aware Architecture for Smart Healthcare Systems", IEEE INTERNET OF THINGS JOURNAL, VOL. 2, NO. 6, DECEMBER 2015.

14. https://www.politesi.polimi.it/bitstream/ 10589/126134/ 3/thesis.pdf

15. https://www.ibm.com/developerworks/library/iot-lp101connectivity-network-protocols/index.html.

\section{BIOGRAPHIES}

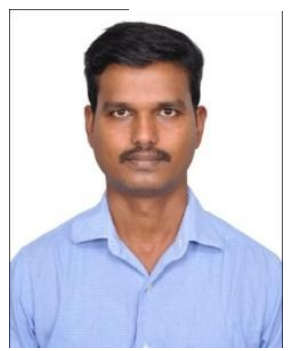

R. Mohandas received Graduation and Post-Graduation Degrees in Computer Science \& Engineering from Anna University Chennai in the year 2005 and 2007 respectively. $\mathrm{He}$ is currently pursuing Ph.D. in the area of Internet of Things (IoT) at Hindustan Institute of Technology and Science, Chennai India,. He is also working as Teaching Faculty in Computer Science Engineering Department at Hindustan Institute of Technology and Science, Chennai India. His area of interest includes IoT, Machine Learning, Computer Networks and Computer Architecture.

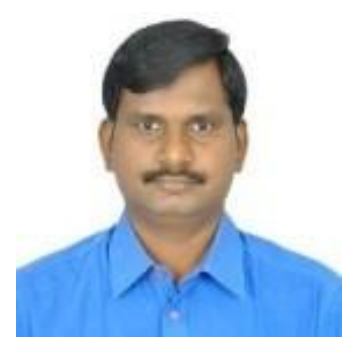

Dr.D. John Aravindhar received the $\mathrm{PhD}$ degree from Hindustan Institute of Technology and Science, Chennai, India. Currently he is working as Professor and Heading the Machine Intelligence and Data Analysis Research Centre (MIDARC) in School of Computing Sciences at Hindustan Institute of Technology and Science, Chennai. His main research interest includes Data mining and Data warehousing, Data Science, Machine learning and IoT.

D. Praveen Kumar received Graduation Degree in Computer Science \& Engineering from Anna University Chennai in the year 2006 and completed his M.Tech post-graduation in Software Engineering at Hindustan Institute of Technology and Science, Chennai. He is currently working as Assistant Professor in CSE Department at Hindustan Institute of Technology and Science, Chennai India. His area of interest includes Software Engineering, Web Technology and Java Programming. 